\title{
Involuntary orienting of attention to a sound desynchronizes the occipital alpha rhythm and improves visual perception
}

\author{
Wenfeng Feng ${ }^{1}$, Viola S. Störmer ${ }^{2,3}$, Antigona Martinez ${ }^{4,5}$, John J. McDonald ${ }^{6}$ and Steven A. \\ Hillyard $^{4}$
}

1Department of Psychology, School of Education, SooChow University, Suzhou, Jiangsu 215123, China,

2Harvard University, Vision Sciences Laboratory, Cambridge, Massachusetts 02138,

3Department of Psychology, University of California, San Diego, La Jolla, California 92093

4Department of Neurosciences, University of California San Diego, La Jolla, California 92093

5Nathan Kline Institute for Psychiatric Research, Orangeburg, New York 10962, and

6Department of Psychology, Simon Fraser University, British Columbia, V5A 1S6, Canada,

Correspondence should be addressed to W.F.F. (fengwfly@gmail.com) 


\begin{abstract}
Directing attention voluntarily to the location of a visual target results in an amplitude reduction (desynchronization) of the occipital alpha rhythm $(8-14 \mathrm{~Hz})$, which is predictive of improved perceptual processing of the target. Here we investigated whether modulations of the occipital alpha rhythm triggered by the involuntary orienting of attention to a salient but spatially non-predictive sound would similarly influence perception of a subsequent visual target. Target discrimination was more accurate when a sound preceded the target at the same location (validly cued trials) than when the sound was on the side opposite to the target (invalidly cued trials). This behavioral effect was accompanied by a sound-induced desynchronization of the alpha rhythm over the lateral occipital scalp. The magnitude of alpha desynchronization over the hemisphere contralateral to the sound predicted correct discriminations of validly cued targets but not of invalidly cued targets. These results support the conclusion that cue-induced alpha desynchronization over the occipital cortex is a manifestation of a general priming mechanism that improves visual processing and that this mechanism can be activated either by the voluntary or involuntary orienting of attention. Further, the observed pattern of alpha modulations preceding correct and incorrect discriminations of valid and invalid targets suggests that involuntary orienting to the non-predictive sound has a rapid and purely facilitatory influence on processing targets on the cued side, with no inhibitory influence on targets on the opposite side.
\end{abstract}

KEY WORDS: alpha oscillation; desynchronization; involuntary attention; voluntary attention; crossmodal cueing; visual discrimination 


\section{INTRODUCTION}

Attending to a stimulus in one modality facilitates the processing of stimuli in other modalities that occur at the location of the attended stimulus (Calvert et al., 2004; Murray \& Wallace, 2012). Such cross-modal facilitation has been observed not only when observers voluntarily direct attention in response to an instructional cue (e.g., a directional arrow) but also when a salient, but otherwise irrelevant, environmental event attracts attention to its location involuntarily (McDonald et al., 2012, 2013a; McDonald \& Ward, 2000; Spence \& Driver, 2004). For example, a sudden noise burst to the left or right of the midline can facilitate speeded responses to nearby visual targets (McDonald \& Ward, 2000; Spence \& Driver, 1997) and enhance the perceptual processing of visual targets at the cued location (Dufour, 1999; Feng et al., 2014; McDonald et al., 2000; McDonald et al., 2005; Störmer et al., 2009), even when the noise burst does not predict the target's location.

Recently, McDonald and colleagues (McDonald et al., 2013b) found that non-predictive auditory cues elicit a slow event-related potential (ERP) shift with an amplitude maximum over the visual cortex contralateral to the side of the sound. The magnitude of this auditory-evoked contralateral occipital positivity (termed the ACOP) was found to predict the perceived contrast of a subsequent visual target (McDonald et al., 2013b) and the accuracy of its discrimination (Feng et al., 2014). The ACOP closely resembles slow-wave ERP activity triggered by voluntary shifts of visual attention (in particular, the late directing attention positivity or LDAP; Harter et al., 1989; Hopf \& Mangun, 2000; McDonald et al., 2013b; Nobre et al., 2000), except that the ACOP is elicited considerably earlier in time. Thus, it was proposed that the ACOP is a neural signature of the involuntary, rapid orienting of visual attention to the location of a salient sound (Hillyard et al., 2016; McDonald et al., 2013a, 2013b). Matusz, Retsa, and Murray (2016) reported that infrequent auditory deviants delivered via earphones elicit the ACOP under 
passive-listening conditions, but only when location of the deviant is unpredictable. These results suggest that the cross-modal activation of visual cortex is reflexive but not entirely automatic (i.e., it is not obligatory).

In addition to slow-wave ERP activity over the posterior scalp, the voluntary orienting of visual-spatial attention is accompanied by strong modulations of the ongoing occipital alpha rhythm $(8-14 \mathrm{~Hz})$ in the cue-target interval. In a widely studied spatial attention paradigm, attention is directed to the left or right visual half-field by a symbolic cue (e.g., a central arrow), which is followed by a target stimulus to be detected or discriminated in either the cued or the uncued hemifield. Alpha power measured in the cue-target interval is typically decreased over the hemisphere contralateral to the attended side, with a lesser decrease or even an increase over the hemisphere ipsilateral to the attended side (for reviews, see Banerjee et al., 2011; Foxe \& Snyder, 2011; Hanslmayr et al., 2011; Ikkai et al., 2016; Jensen \& Mazaheri, 2010; Klimesch, 2012; Payne \& Sekuler, 2014; Thut et al., 2012). The decreased (desynchronized) alpha rhythm over the contralateral hemisphere has been linked with improved processing of the attended-field stimulus, whereas the relative increase in ipsilateral alpha power has been considered a sign of the active suppression of distracting inputs from the unattended visual half-field (Green \& McDonald, 2010; Kelly et al., 2006; Händel et al., 2011; Okazaki et al., 2014; Payne et al., 2013; Rihs et al., 2007, 2009; Sauseng et al., 2005; Worden et al., 2000). Consistent with these findings, decreased alpha amplitudes have been correlated with improved perceptual processing of visual events at voluntarily attended locations in a wide variety of tasks (e.g., Ergenoglu et al., 2004; Gould et al., 2011; Hanslmayr et al., 2007; Kelly et al., 2009; Mazaheri et al., 2014; Romei et al., 2008; Thut et al., 2006, 2012; Trenner et al., 2008; van Dijk et al., 2008; Yamagishi et al., 2008). 
While the foregoing studies have demonstrated a strong linkage between the voluntary allocation of visual-spatial attention and modulation of the occipital alpha rhythm, little is known about modulations of the alpha rhythm that accompany the involuntary orienting of attention or whether such modulations have an effect on perceptual processing. Behavioral studies over the past four decades have drawn a fundamental distinction between the voluntary (top-down or endogenous) orienting of attention to a target's location by a symbolic cue and the involuntary (bottom-up, exogenous or automatic) capture of attention by a salient physical event that is not informative regarding the target's location (Briand, 1998; Jonides, 1980; Müller \& Rabbit, 1989; Posner \& Cohen, 1984; reviewed in Wright \& Ward, 2008). Voluntary and involuntary orienting of attention differ in several important properties. Whereas voluntary orienting proceeds slowly and may be sustained over periods of one to several seconds, involuntary orienting is transient, with a rapid onset and a transition into an inhibitory phase (inhibition of return) after several hundred milliseconds. Involuntary attention is also less sensitive to concurrent task interference and to the predictability of target location by the cue. Neuroimaging studies indicate that these two forms of attention are mediated by different neural networks (Corbetta \& Shulman, 2002; Kincade et al., 2005; Mayers et al., 2004; reviewed in Santangelo et al., 2009).

Previous studies have reported that the involuntary orienting of attention to a lateralized vibrotactile (Trenner et al., 2008) or auditory (Mlynarski et al., 2014; Störmer et al., 2016) stimulus is associated with a sharp decrease of the occipital alpha rhythm that is more pronounced over the hemisphere contralateral to the stimulus location. However, it remains unclear whether such involuntary modulations of the occipital alpha rhythm have any impact upon perceptual processing of visual events. The present study investigates this question in the cross-modal cueing paradigm previously described by Feng et al. (2014), in which a peripheral sound cue was followed after $400 \mathrm{~ms}$ by a masked letter (T or L) presented unpredictably to the 
same or opposite side as the sound (see Fig. 1). One advantage of using an auditory (rather than a visual) cue to examine attention effects on the occipital alpha rhythm is the absence of a visual evoked potential elicited by the cue, which typically contains alpha-band activity that may confound measurements of attention-related alpha modulations in the cue-target interval. To preview the present results, we found that contralateral alpha desynchronization associated with the involuntary orienting of attention to the sound was highly predictive of accurate visual perception, but only when the targets appeared on the same side as the sound (i.e., when targets were validly cued).

\section{MATERIALS \& METHODS}

Participants. Sixteen subjects (10 female; mean age of 22.5 years) volunteered to participate in the experiment. All subjects provided informed written consent as approved by the Human Research Protections Program of the University of California San Diego. All participants reported normal or corrected to normal vision and normal hearing.

Stimuli and procedure. The details of stimuli and procedure for this study were described previously (Feng et al., 2014). Here we report an extended analysis of the data obtained by Feng et al. (2014) that examines modulations of the alpha rhythm and their relationships to the concurrently recorded slow potential (ACOP) changes and discrimination performance measures that were reported previously.

The experiment was conducted in a dimly lit, electrically shielded and sound-attenuated chamber. Visual stimuli were presented on a 27-in computer monitor, and auditory stimuli (50015,000 Hz pink noise, $78 \mathrm{~dB}$ SPL, $83 \mathrm{~ms}$ duration with $5 \mathrm{~ms}$ rise and fall ramps) were delivered by a pair of free-field speakers positioned at the left and right sides of the monitor (Fig. 1). On each trial a spatially non-predictive auditory cue (pink noise burst) was presented randomly from 
one of the speakers (50\% left; 50\% right). Following this auditory cue with a stimulus-onset asynchrony (SOA) of $400 \mathrm{~ms}$, the target letter (either "T" or "L", height 2.4 degrees) was presented for $50 \mathrm{~ms}$ at an eccentricity of 17.5 degrees lateral to fixation on either the same side as the sound cue (validly cued) or the opposite side (invalidly cued) on $57 \%$ of the trials (valid $28.5 \%$, invalid $28.5 \%$. in random order). The target letter was immediately masked by a grid of superimposed lines ( 7 by 7 degrees of visual angle) that was presented for $100 \mathrm{~ms}$. The participants were asked to discriminate the letter target by pressing one of two buttons (counterbalanced for " $\mathrm{T}$ " and "L" across participants) on a game pad device. The instructions emphasized accuracy rather than speed of response. No responses were required when letters were absent on mask-only "catch" trials $(28.5 \%)$ where the grid mask was presented to the left or right side at random without any preceding letter, and cue-only trials (14.5\%) where no visual stimulus was presented after the sound cue. All these types of trials were presented in random order with inter-trial intervals ranging from 1200 to $1450 \mathrm{~ms}$. During the experiment, participants were required to maintain their eyes fixated on a central cross $(0.7$ by 0.7 degree of visual angle). The experiment consisted of 15 blocks of 126 trials each. Discrimination accuracy was quantified in terms of percent correct choices and in terms of sensitivity (d') for the T vs. L discrimination on the letter-present trials.

Electrophysiological recording and analysis. The EEG was recorded continuously from 60 scalp sites using a modified 10-10 system montage (McDonald et al., 2003). Horizontal eye movements were recorded bipolarly via electrodes at the left and right outer canthi (Horizontal Electro-Oculogram: HEOG). Vertical eye movements and blinks were monitored by an electrode below the left eye (VEOG). The electrode impedances were kept below $5 \mathrm{k} \Omega$. The EEG and EOG signals were digitized at $250 \mathrm{~Hz}$, amplified with a gain of 10,000, and filtered with an 
amplifier band pass of 0.01 to $80 \mathrm{~Hz}$. The right mastoid electrode served as the reference during data acquisition.

The EEG signals were analyzed separately in the time domain (as averaged ERPs) and in the time-frequency domain using wavelets. For both analyses, the EEG signals were time-locked to the auditory stimulus onset, and the EEG data recorded on the letter-present trials were segregated in relation to discrimination accuracy. Thus, the EEG and ERPs were averaged separately for trials with validly and invalidly cued target locations and with correct and incorrect target discrimination responses. Epochs contaminated by eye movements, eye blinks, muscle activity, or amplifier blocking were rejected. For analysis purposes, the EEG data were collapsed across auditory stimulus location (left, right) and hemisphere of recording (left, right) to obtain EEG epochs and ERPs recorded from the contralateral and ipsilateral hemispheres with respect to the auditory stimulus location (e.g., see Fig. 2A). Specifically, in order to obtain the contralateral EEG response to the auditory cue the EEG activity recorded from the right hemisphere in response to the left sounds was averaged together with the EEG activity recorded from the left hemisphere in response to the right sounds. This collapsing of the EEG data was justified by first showing that the contralateral/ipsilateral amplitude ratios of the relevant EEG measures did not differ significantly between the left cue and right cue conditions.

In the ERP analysis, the EEG signals were averaged in $500 \mathrm{~ms}$ epochs with respect to a $100 \mathrm{~ms}$ pre-stimulus baseline. The averaged waveforms were digitally low-pass filtered ( $-3 \mathrm{~dB}$ cutoff at $25 \mathrm{~Hz}$ ) and re-referenced to the average of the left and right mastoids. The ACOP component was measured as the mean contralateral minus ipsilateral amplitude averaged over 6 pairs of posterior electrode sites (P7/P8, PO3/PO4, PO7/PO8, O1/O2, I3/I4, I5/I6) within the time windows of 200-300 and 300-400 ms post-stimulus onset with respect to a $100 \mathrm{~ms}$ prestimulus baseline. 
Time-frequency analysis was carried out using EEGLAB (Delorme \& Makeig, 2004) and ERPLAB (Lopez-Calderon \& Luck, 2014) toolboxes. EEG data were segmented into epochs spanning -1500 to $+1500 \mathrm{~ms}$ with respect to onset of the auditory cue, re-referenced to the average of the left and right mastoids, and analyzed on a single-trial basis via convolution with complex Morlet wavelets prior to averaging. Spectral amplitudes for each frequency were estimated using 3-cycle wavelets for each electrode, trial and time point (every $4 \mathrm{~ms}$ ). Single trial spectral amplitudes for each frequency were then averaged across trials over -400 to $500 \mathrm{~ms}$ epochs with the baseline set at -400 to -200 ms. Modulations of alpha amplitude were measured as the change with respect to baseline averaged over the frequencies of $8-14 \mathrm{~Hz}$ and over the same electrodes and the same time intervals (200-300 and 300-400 ms after the cue) as for the ERP analysis. In other words, the alpha amplitude calculated over the -400 to $-200 \mathrm{~ms}$ baseline was subtracted from the alpha amplitudes calculated over the $200-300$ and $300-400$ ms post-cue intervals to obtain the measures of alpha desynchronization produced by the cue.

The ERP amplitudes and alpha modulations on letter-present trials in the 200-300 ms and 300-400 ms intervals after the cue were analyzed separately in 3-way repeated-measures ANOVAs with factors of hemisphere (contralateral/ipsilateral with respect to side of cue), cue validity (valid/invalid cue-target relationship) and letter discrimination accuracy (correct/incorrect). In cases of significant 3-way interactions, follow-up analyses were carried out in which the ERPs and alpha modulations were quantified as the differences between the amplitudes at contralateral minus ipsilateral homologous sites for each of the four types of trials (valid/invalid $\times$ correct/incorrect). The resulting ERP and alpha-band difference amplitudes were separately analyzed with specific contrasts between correct and incorrect trials for each validity condition. To investigate whether the alpha rhythm showed relative increases or decreases in the hemispheres ipsilateral and contralateral to the sound across the different trial types, a validity $\times$ 
accuracy 2-way ANOVA was also carried out for each hemisphere separately for the alpha amplitude modulations. Further, to investigate the relationship between sound-induced occipital activities observed in the time domain (ERPs/ACOP) and the time-frequency domain (alpha modulations), between-subject correlations of ACOP amplitudes with lateralized alpha desynchronization (contralateral-minus-ipsilateral) amplitudes were calculated for each type of trial.

To examine whether the sounds induced phase locking of the alpha rhythm in association with its desynchronization (Mercier et al., 2013; Romei et al., 2012), the phase locking index (inter-trial phase coherence measure) was calculated for alpha-band activity (8-14 Hz) for posterior electrode sites where alpha desynchronization was maximal. This measure of inter-trial phase consistency was calculated using the same complex wavelet decompositions as for the amplitude analyses, which were normalized on every trial by its absolute value and then averaged across all trials of the same type.

Topographical mapping and source localization. The topographical scalp distributions were plotted for the ACOP and for the lateralized alpha desynchronization obtained by subtracting the ipsilateral from the contralateral alpha amplitudes. These contralateral minus ipsilateral ACOP and alpha amplitude distributions (grand averaged over all subjects) were projected to the right hemisphere of each topographical map (e.g., see Fig. 2, right column). The method of McCarthy and Wood (1985) was used to statistically compare the topographical distributions of the ACOP and the lateralized alpha desynchronization. The McCarthy-Wood procedure compares two topographical scalp distributions (conditions) by first normalizing their amplitudes over the array of scalp electrodes and then performing an ANOVA to see if there is an interaction between scalp site and condition. A significant interaction is interpreted as a difference in topography. 
The anatomical sources of the ACOP and lateralized alpha desynchronization elicited by the sound (each quantified as a contralateral minus ipsilateral amplitude difference) were estimated within the time interval of 300-400 ms after sound onset from the grand-averaged topographies on letter-present trials by means of distributed linear inverse solutions based on a local autoregressive average (LAURA) (Grave de Peralta Menendez et al., 2004). LAURA estimates 3D current density distributions using a realistic head model with a solution space of 4024 nodes equally distributed within the gray matter of the average template brain of the Montreal Neurological Institute. LAURA analyses were implemented using Cartool software. The resulting source estimations were transformed into the standardized coordinate system of Talairach and Tournoux (1988) and projected onto a structural brain image supplied by MRIcro (Rorden \& Brett, 2000) using the AFNI (Analysis of Functional NeuroImaging) software (Cox, 1996).

\section{RESULTS}

\section{Sounds Improve Discrimination of Visual Targets.}

Participants were asked to discriminate lateralized visual letters (T vs. L) that were preceded by a spatially non-predictive lateralized sound (i.e., the auditory cue) and were followed immediately by a mask (see Fig. 1). As reported previously (Feng et al., 2014), the behavioral results showed that the non-predictive auditory cue improved the discrimination of co-localized visual letters, with higher accuracy, higher d-primes and shorter reaction times in the validly cued condition than in the invalidly cued condition (accuracy: valid $72.5 \% \pm 12.3$, invalid $69.6 \% \pm 12.5$; d-prime: valid $1.57 \pm 0.70$, invalid $1.44 \pm 0.67$; reaction time: valid $545 \pm 65$ $\mathrm{ms}$, invalid $553 \pm 64 \mathrm{~ms}$; all $p$ 's $<0.001$ by one-way analyses of variance). 


\section{Sounds Elicit ACOP and Lateralized Alpha Desynchronization that Predict Target Discrimination Accuracy.}

On a random $14.5 \%$ of the trials, no visual stimuli were presented. On these cue-only trials, the non-predictive auditory cue elicited a contralateral ERP positivity (ACOP) that was paralleled by a lateralized amplitude decrease of the ongoing alpha-band EEG oscillations (i.e., alpha desynchronization; Fig. 2). The ACOP and the concurrent alpha desynchronization began at about the same time (around $200 \mathrm{~ms}$ after the sound cue), and both were of greater magnitude over the hemisphere contralateral to the side of the cue. The scalp topographies of ACOP and lateralized alpha desynchronization were also very similar, with amplitude maxima over the lateral occipital scalp (Fig. 2 A \& B). Indeed, statistical comparisons did not reveal any reliable differences between the two scalp distributions.

-----------------Insert Figures 1 and 2 about here------------------

On those trials where visual targets were presented, the ACOP amplitude strongly predicted the behavioral outcome of the letter discrimination task, as reported previously (Feng et al., 2014). When the letter targets were validly cued, the ACOP amplitude (contralateral minus ipsilateral difference averaged over the intervals $200-300 \mathrm{~ms}$ and $300-400 \mathrm{~ms}$ following the cue) was larger preceding correct than incorrect discriminations [200-300 ms: $F(1,15)=5.43$, $\mathrm{p}<0.05 ; 300-400$ ms: $\mathrm{F}(1,15)=10.80, \mathrm{p}<0.005$ ] (Fig. 3A \& B, Fig. 4A \& B, left columns). In contrast, on invalidly cued trials the ACOP was of intermediate amplitude and did not differ significantly between trials with correct and incorrect discriminations [200-300ms: $F(1,15)=$ $0.32, \mathrm{p}=0.58 ; 300-400 \mathrm{~ms}: \mathrm{F}(1,15)=0.24, \mathrm{p}=0.63]$ (Fig. 3C \& D, Fig. 4C \& D, left columns). These effects resulted in a 3-way interaction of hemisphere $\mathrm{x}$ validity $\mathrm{x}$ accuracy in the ANOVA 
of ACOP amplitude within both time intervals [200-300 ms: $F(1,15)=6.14$, p < 0.05; 300-400 ms: $F(1,15)=11.12, p<0.005$ ] (Fig. 5A \& B, left bars). Specific contrasts showed that a highly significant ACOP was elicited on validly cued correct trials [contralateral vs ipsilateral, 200-300 ms: $F(1,15)=21.40, p<0.0005 ; 300-400 m s: F(1,15)=68.64, p<0.000001]$ (Figs. 3A and 4A, left) but not on validly cued incorrect trials [contralateral vs ipsilateral, 200-300 ms: F(1,15) = $0.16, \mathrm{p}=0.68 ; 300-400 \mathrm{~ms} F(1,15)=0.12, \mathrm{p}=0.73$ ] (Figs. 3B and 4B, left). For invalidly cued targets, however, a significant ACOP was present both on correct trials [contralateral vs ipsilateral, 200-300 ms: $F(1,15)=9.36, p<0.01 ; 300-400 m s: F(1,15)=12.04, p<0.005]$ (Figs. $3 \mathrm{C}$ and $4 \mathrm{C}$, left) and on incorrect trials [contralateral vs ipsilateral, 200-300 ms: $\mathrm{F}(1,15)=15.61$, $\mathrm{p}<0.005 ; 300-400 \mathrm{~ms}: \mathrm{F}(1,15)=19.03, \mathrm{p}<0.001$ ] (Figs. 3D and 4D, left).

-Insert Figure 3 and 4 about here

As shown in the right column of Fig. 3, the amplitude modulations of lateralized alpha desynchronization (contralateral minus ipsilateral alpha amplitude, averaged over $8-14 \mathrm{~Hz}$ in the intervals of $200-300 \mathrm{~ms}$ and $300-400 \mathrm{~ms}$ following the auditory cue) closely paralleled those of the ACOP. As with the ACOP, the 3-way interaction of hemisphere $\mathrm{x}$ validity $\mathrm{x}$ accuracy was significant for the alpha desynchronization measure within $200-300 \mathrm{~ms}$ interval $[\mathrm{F}(1,15)=5.16$, $\mathrm{p}<0.05]$ and $300-400 \mathrm{~ms}$ interval $[\mathrm{F}(1,15)=12.14, \mathrm{p}<0.005]$. On validly cued trials (Fig. 3, A\&B, Fig. 4, A\&B, right columns), the lateralized alpha desynchronization (contralateral minus ipsilateral amplitude) was reliably larger preceding correct than incorrect discriminations within both time intervals $[200-300 \mathrm{~ms}: \mathrm{F}(1,15)=14.87, \mathrm{p}<0.01 ; 300-400 \mathrm{~ms}: \mathrm{F}(1,15)=20.62, \mathrm{p}<$ 0.001 ], whereas on invalidly cued trials (Fig. 3C \& D, Fig. 4C \& D, right columns) the desynchronization was of intermediate amplitude and did not differ significantly between trials 
having correct and incorrect discriminations [200-300 ms \& 300-400 ms: $F^{\prime} s(1,15)<1, p$ 's $>$ 0.05]. Specific contrasts showed that the lateralized alpha asymmetry (over 200-300 ms and $300-400 \mathrm{~ms}$ ) was significant on valid correct trials [larger alpha desynchronization over the contralateral vs ipsilateral hemisphere, $200-300 \mathrm{~ms}: \mathrm{F}(1,15)=60.20, \mathrm{p}<10^{-5} ; 300-400 \mathrm{~ms}$ : $\left.F(1,15)=100.84, p<10^{-6}\right]$, but not on valid incorrect trials $[200-300 \mathrm{~ms}: F(1,15)=0.10, p=0.76$; 300-400ms: $F(1,15)=0.77, p=0.39$ ] (Figs. 3, A\&B and 4 A\&B, right). However, for invalidly cued trials, significant lateralized alpha desynchronization was found for both correct trials [contralateral vs ipsilateral, 200-300 ms: $\mathrm{F}(1,15)=11.18$, $\mathrm{p}<0.005 ; 300-400 \mathrm{~ms}: \mathrm{F}(1,15)=5.19$, $\mathrm{p}<0.05$ ] and incorrect trials [contralateral vs ipsilateral, 200-300ms $\mathrm{F}(1,15)=53.65, \mathrm{p}<0.00001$; 300-400ms: $F(1,15)=26.99, p<0.001]$ (Figs. 3C\&D and 4C \& D).

These significant modulations of alpha amplitude following the auditory cue in the different conditions were not accompanied by any consistent differences in phase-resetting of the alpha rhythm. Time-frequency plots of the phase-locking index derived from the wavelet analysis are shown in Supplementary Fig. 1 for the different types of trials. Statistical analysis found no significant differences in alpha-band phase-locking between the different trial types in the 200-400 ms range (see Supplementary Fig. 1 legend).

As shown in Fig. 5, the ACOP and lateralized alpha desynchronization displayed very similar amplitude variations as a function of trial type (validity $\mathrm{x}$ accuracy) over both the 200300 and 300-400 ms intervals. To further investigate the relationship between ACOP and alpha desynchronization, between-subject correlations of ACOP amplitude with lateralized alpha desynchronization (contralateral minus ipsilateral amplitude over 200-400 ms post-cue) were calculated for each of the different types of trials. The results indicate that participants who showed larger ACOPs also exhibited stronger alpha desynchronization for all trial types where these effects were present: valid correct trials $(r=-0.54, p<0.05)$; invalid correct trials $(r=-0.68$, 
$\mathrm{p}<0.005)$; and invalid incorrect trials $(\mathrm{r}=-0.62, \mathrm{p}<0.05)$. Scatter plots of these correlations are shown in Fig. 6. No significant correlation was found for the valid incorrect trials $(r=0.27, p=$ 0.31), in which no appreciable ACOP or alpha desynchronization was present.

-Insert Figure 5 about here

Insert Figure 6 about here

\section{Additional Controls for Temporal Specificity of Alpha Modulations}

Since the three-cycle wavelet used in this analysis extends over $375 \mathrm{~ms}$ at $8 \mathrm{~Hz}$, it is conceivable that alpha amplitude differences in the pre-cue baseline period (-400 to $-200 \mathrm{~ms})$ might have influenced the measurements of alpha amplitude in the post-cue period. To examine this possibility of baseline differences, the raw alpha band (8-14 Hz) amplitudes in the $200 \mathrm{~ms}$ baseline interval preceding the different types of trials were subjected to a 3-way ANOVA with factors of validity (valid vs. invalid) x accuracy (correct vs. incorrect) and hemisphere (contralateral/ipsilateral with respect to the side of cue. There were no significant main effects of validity, accuracy or hemisphere [validity $\mathrm{F}(1,15)=0.445, \mathrm{p}=0.515$; accuracy $\mathrm{F}(1,15)=0.362$, $\mathrm{p}=0.556$; hemisphere $\mathrm{F}(1,15)=0.010, \mathrm{p}=0.922]$. Nor was the validity $\mathrm{x}$ accuracy $\mathrm{x}$ hemisphere 3-way interaction significant $[F(1,15)=1.156, \mathrm{p}=0.299]$. Thus, the differences in alpha amplitudes in the cue-target interval between the different trial types (Figs. 3-5) could not be attributed to alpha amplitude differences in the pre-cue baseline period.

To further verify the temporal specificity of the observed alpha modulations, the alpha amplitudes in the $200-300 \mathrm{~ms}$ and $300-400 \mathrm{~ms}$ post-cue intervals were reanalyzed over a narrower range of the alpha band $(10-14 \mathrm{~Hz})$ for which the wavelet would have a shorter duration (300 ms or less). The same pattern of results was found as with the $8-14 \mathrm{~Hz}$ 
measurements (see Fig. S2); the validity $\times$ accuracy $\times$ hemisphere 3 -way interactions were significant for both $200-300 \mathrm{~ms}$ interval $[\mathrm{F}(1,15)=7.931, \mathrm{p}<0.02]$ and $300-400 \mathrm{~ms}$ interval $[F(1,15)=11.978, \mathrm{p}<0.005]$. Further analysis showed that the lateralized alpha desynchronization (contralateral minus ipsilateral amplitude) was significantly larger for valid correct trials than valid incorrect trials within both time intervals [200-300ms: $\mathrm{F}(1,15)=10.643$, $\mathrm{p}<0.01 ; 300-400 \mathrm{~ms}: \mathrm{F}(1,15)=18.680, \mathrm{p}<0.001]$, whereas the lateralized alpha desynchronization did not differ between invalid correct trials and invalid incorrect trials in these time intervals $[200-300 \mathrm{~ms}: F(1,15)=0.179, p=0.678 ; 300-400 \mathrm{~ms}: F(1,15)=0.023, p=$

0.882 ]. The close correspondence between the patterns of alpha modulation measured over 10$14 \mathrm{~Hz}$ and 8-14 Hz (compare Figs. 5 and S2) for both the 200-300 ms and 300-400 ms intervals provides strong evidence that the differences in alpha desynchronization between the types of trials arise from neural activity within these intervals and not from a "leakage" of activity occurring during the pre-cue or post-target intervals.

\section{Sounds Elicit Occipital Alpha Desynchronization Over both Contralateral and Ipsilateral Hemispheres.}

The magnitude of the auditory-induced alpha desynchronization was also examined separately over the hemispheres contralateral and ipsilateral to the side of the sound. As shown in Fig. 7, the alpha activity was significantly decreased with respect to the pre-cue baseline over both hemispheres on all types of trials (all $t$ 's $<-3.5$, all $p$ 's $<0.01$ ). Two-way ANOVAs with factors of validity and accuracy were carried out on the amplitude of this alpha desynchronization for each hemisphere separately. There were no significant main effects of validity or accuracy for either hemisphere, and no significant validity $\mathrm{x}$ accuracy interaction for the hemisphere ipsilateral to the sound $[F(1,15)=0.94, p>0.05]$. For the hemisphere 
contralateral to the sound, however, the validity $\mathrm{x}$ accuracy interaction was significant $[\mathrm{F}(1,15)=$ $4.55, \mathrm{p}<0.05]$, and further analysis showed that the contralateral alpha desynchronization was greater on correct than incorrect trials for validly cued targets $[\mathrm{F}(1,15)=10.61, \mathrm{p}<0.01]$, but not for invalidly cued targets $[F(1,15)=0.41, p>0.05]$. Thus, the lateral asymmetry of the soundinduced alpha desynchronization, which correlated strongly with discrimination performance, was primarily determined by the level of desynchronization recorded over the contralateral hemisphere.

Insert Figure 7 about here-

\section{Sound-Elicited Alpha Desynchronization and ACOP Show Similar Topographies and Neural Sources.}

The scalp topographies of the lateralized alpha desynchronization and the ACOP amplitudes both had a lateral occipital focus on the valid correct trials (Fig. 4A) as well as on the invalid correct and incorrect trials (Fig. 4C \& 4D). Statistical comparisons of the scalp topographies of the ACOP and lateralized alpha desynchronization using the McCarthy and Wood (1985) procedure showed no significant differences in their distributions for any of these trial types [electrode site $\times$ type of response (ACOP vs alpha asymmetry) interaction, all $\left.p^{\prime} \mathrm{s}>0.05\right]$. On the valid incorrect trials both the ACOP and alpha desynchronization were absent (Fig. 3B, Fig. 4B).

Based on their grand averaged scalp topographies, the neural sources of the ACOP and the lateralized alpha desynchronization were estimated using the LAURA algorithm within the time window of $300-400 \mathrm{~ms}$ after sound onset. The estimated sources were transformed into the standardized coordinate system of Talairach and Tournoux (1988) and projected onto a structural 
brain image (Rorden \& Brett, 2000) (Fig. 8). Consistent with the similarity of the topographical distributions, LAURA analysis revealed the maximal regions of current density for ACOP and lateralized alpha desynchronization to be in close proximity for the different types of trials: valid correct (ACOP: $x=26, y=-79, z=-8$; alpha: $x=34, y=-72, z=-10)$, invalid correct (ACOP: $x=28$, $\mathrm{y}=-76, \mathrm{z}=-6$; alpha: $\mathrm{x}=31, \mathrm{y}=-78, \mathrm{z}=-9$ ) and invalid incorrect (ACOP: $\mathrm{x}=33, \mathrm{y}=-70, \mathrm{z}=-8$; alpha: $x=32, y=-76, z=-8)$. These source localizations were situated in the ventrolateral occipital cortex (in or near Brodmann's area 19) and are consistent with the source localizations of ACOP and alpha desynchronization reported previously (Feng et al., 2014; McDonald et al., 2013b; Störmer et al., 2016). Insert Figure 8 about here-

\section{DISCUSSION}

The present study examined modulations of the occipital alpha rhythm $(8-14 \mathrm{~Hz})$ in the cross-modal cueing paradigm previously reported by Feng et al. (2014), wherein a lateralized sound cue was followed after $400 \mathrm{~ms}$ by a masked letter target (T or L) presented unpredictably to the same or opposite side as the sound. We found that the alpha rhythm was decreased in amplitude (i.e., desynchronized) following the auditory cue, with greater desynchronization over the hemisphere contralateral to the side of the cue. The magnitude of this contralateral alpha desynchronization was strongly predictive of visual target discrimination accuracy on validly cued trials (i.e., when the target by chance occurred at the same location as the non-predictive auditory cue). Specifically, contralateral alpha desynchronization was present on validly cued trials when subjects correctly discriminated the visual target, but was absent preceding incorrect discriminations. In contrast, on invalidly cued trials (i.e., when the target occurred at the location opposite to that of the non-predictive auditory cue) the lateralized alpha desynchronization was 
of intermediate amplitude and did not differ in amplitude between correct and incorrect discriminations. We hypothesize that these lateralized modulations of the alpha rhythm and the accompanying ACOP slow potential are neural manifestations of the involuntary orienting of spatial attention to the sound's location that facilitates the perceptual processing of visual stimuli at the location of the sound. Such spatially specific facilitation would enhance accuracy of visual discrimination on valid trials without necessarily affecting accuracy of visual discrimination on invalid trials.

The pattern of alpha modulations during involuntary orienting to a non-predictive (exogenous) auditory cue has definite similarities to the alpha modulations that have been reported to follow a symbolic (endogenous) cue that instructs a participant to attend voluntarily to either the left or right visual field (for reviews, see: Foxe \& Snyder, 2011; Hanslmayr et al., 2011; Jensen \& Mazaheri, 2010; Klimesch, 2012; Payne \& Sekuler, 2014; Thut et al., 2012). Both voluntary and involuntary orienting of attention produce a greater desynchronization of the occipital alpha rhythm over the hemisphere contralateral to the cue or cued location. Most importantly, the degree of lateralized alpha desynchronization that accompanied involuntary orienting to a sound in the present study was strongly predictive of the perceptual accuracy of the ensuing visual target, as has been reported in many previous studies of alpha modulations that accompany the voluntary orienting of attention (see Introduction). These similarities suggest that modulations of the occipital alpha rhythm reflect a general priming mechanism that biases perceptual processing of visual stimuli and can be initiated either by the voluntary focusing of attention under top-down control or by the involuntary capture of attention by a salient stimulus.

Besides the aforementioned similarities, there are also distinct differences between the alpha modulations elicited by the spatially non-predictive sound and those reported to occur in association with the voluntary cueing of attention to the left or right visual field. First, the 
involuntary alpha desynchronization takes place earlier, starting at around $200 \mathrm{~ms}$, while the alpha modulations associated with voluntary shifts of attention typically occur at a longer latency (Green \& McDonald, 2010; Händel et al., 2011; Kelly et al., 2009; Rihs et al., 2007, 2009; Sauseng et al., 2005; Snyder \& Foxe, 2010; Thut et al., 2006; Worden et al., 2000; Yamagishi et al., 2005). This difference in timing is consistent with well-known observations that the influence of exogenous (involuntary) cueing on behavior and perception occurs at shorter cue-target intervals (100-200 ms) than endogenous (voluntary) cueing effects, which typically begin at intervals of 300-600 ms (e.g., Cheal \& Lyon, 1991; Jonides, 1980; Nakayama \& Mackeben, 1989; reviewed in Wright \& Ward, 2008). Second, in many studies where attention was directed voluntarily to the left or right visual field, the contralateral decrease in alpha amplitude was accompanied by an increase in alpha amplitude over the hemisphere ipsilateral to the attended field (e.g., Foxe \& Snyder, 2011; Green \& McDonald, 2010; Händel et al., 2011; Kelly et al., 2006; Okazaki et al., 2014; Payne et al., 2013; Rihs et al., 2007, 2009; Sauseng et al., 2005; Worden et al., 2000). This ipsilateral increase was generally interpreted as a neural sign of the suppression of visual information presented to the unattended half-field. In the present study, however, the non-predictive auditory cue provoked a decrease in alpha-band amplitude over both the ipsilateral and contralateral hemispheres within the $400 \mathrm{~ms}$ cue-target interval, with a lesser decrease observed ipsilaterally. Whether or not the ipsilateral alpha amplitude would actually show an increase relative to the pre-cue baseline with longer cue-target intervals following a non-predictive cue requires further study.

The specific pattern of alpha amplitude modulations observed here following the nonpredictive sound is informative regarding the question of whether visual input was suppressed in the visual field opposite to the sound's location in the $400 \mathrm{~ms}$ interval preceding the visual target. Whereas the amplitude of the contralateral alpha desynchronization (and the contralateral minus 
ipsilateral difference) was strongly predictive of discrimination accuracy of validly cued visual targets, the concurrent ipsilateral desynchronization was of equal magnitude for correct and incorrect trials and was thus not predictive of the accuracy of discriminating invalidly cued targets (see Fig. 7). This suggests that the non-predictive auditory cue enhances visual processing at the cued location (associated with alpha desynchronization over the contralateral hemisphere) without suppressing visual processing in the uncued hemi-field. If such a suppression (associated with a relative increase in alpha amplitude over the ipsilateral hemisphere) had been induced by the auditory cue, we would expect to see a relatively larger ipsilateral alpha increase (and a larger ACOP; Feng et al., 2014) preceding incorrect versus correct discriminations of invalidly cued targets. The absence of such an ipsilateral alpha increase before incorrect discriminations suggests that the cue-induced alpha desynchronization over occipital cortex influences the discriminative processing of visual targets at the cued location but not at the uncued location. These data thus suggest that the involuntary orienting of attention has a purely facilitatory influence on visual processing at the cued location without any inhibitory effect on stimuli at the uncued location, at least within the short (400 ms) cue-target interval studied here.

The present design follows previous studies of involuntary (exogenously cued) attention by using a spatially non-predictive peripheral cue (Wright $\&$ Ward, 2008), but it may be questioned whether some degree of voluntary (endogenous) attention might also have been engaged by the spatially non-predictive auditory cue. This seems unlikely for several reasons. First, the lateralized alpha desynchronization observed here was well-developed over the 200300 ms post-cue interval, whereas alpha modulations associated with voluntary attention typically do not begin until around 400 ms post-cue (Banerjee et al., 2011; Händel et al., 2011; Kelly et al., 2009; Thut et al., 2006;Worden et al., 2000; Yamagishi et al., 2005). Second, a 
similar early alpha desynchronization was recently observed in response to a lateralized auditory stimulus that was completely task-irrelevant and had no spatial or temporal predictive value (Störmer et al., 2016), thus demonstrating the involuntary nature of this lateralized desynchronization effect. Third, voluntarily attending to a cued location typically gives rise to a processing cost on the uncued side and an increase in alpha-band activity contralateral to the uncued location. Had voluntary attention mechanisms been engaged in the current study, such uncued-location costs should have been evident, but were not. Fourth, prior studies have demonstrated that non-predictive peripheral auditory cue can facilitate responses to nearby visual targets even when attention is voluntarily engaged elsewhere in the visual field (Chen et al., 2012; Koelewijn et al., 2009; Santangelo et al., 2009). Thus, it appears that the involuntary, bottom-up capture of visual attention by a salient auditory stimulus is a highly robust phenomenon that can occur regardless of the engagement of voluntary, endogenous attention.

The pattern of alpha desynchronization observed here was closely paralleled by amplitude modulations of the concurrently recorded slow positivity (ACOP). The lateralized alpha desynchronization and the ACOP overlapped in time and had very similar scalp topographies and estimated neural sources in the ventral-lateral extrastriate visual cortex. In addition, both the contralateral alpha desynchronization and the ACOP were similarly associated with visual discrimination performance (see Fig. 5). Furthermore, the magnitude of the lateralized alpha desynchronization and ACOP amplitudes were strongly correlated between participants. These similarities are indicative of a close relationship between these two distinctive measures of cue-induced neural activity. Störmer et al. (2016) also observed a correlation between the sound-induced alpha desynchronization and the ACOP in purely auditory tasks in which no visual stimuli were presented at all. Remarkably, alpha desynchronization and ACOP were concurrently elicited even by sounds that were irrelevant to 
the ongoing task, consistent with our hypothesis that they represent neural events engaged during the involuntary orienting of attention to the salient sounds. As in the present study, Störmer et al. (2016) found that contralateral alpha desynchronization and ACOP showed strong temporal and topographical correspondence, and the magnitudes of both measures were also correlated on a trial by trial basis and between participants. These parallels suggest that ACOP and alpha desynchronization represent different aspects of a common biasing mechanism in visual cortex.

It has been proposed that slow waves (such as the ACOP) may be produced in the averaged ERP by changes in oscillatory brain activity (Mazaheri \& Jensen, 2008). Specifically, if the peaks and troughs of ongoing oscillations were modulated asymmetrically (and were not phase-locked to the cue), these asymmetries could in principle summate to form a slow wave in the ERP elicited by the cue when averaged over many trials (van Dijk et al., 2010). According to this hypothesis the ACOP might be a by-product of the lateralized alpha desynchronization, and both these measures would then reflect the same biasing mechanism in visual cortex. A second possibility would be that the ACOP and alpha desynchronization represent separate biasing mechanisms in visual cortex that are both initiated at the same point in time in response to a common event (such as the orienting of attention to a sound). At present, however, it is not possible to distinguish between the alpha asymmetry hypothesis and the separate mechanisms account because in both cases the data would show a co-variation of alpha-band activity and the ACOP slow potential.

\section{SUMMARY}

The present results show that a salient but spatially non-predictive auditory cue can trigger lateralized changes in the occipital alpha rhythm and further demonstrate that these changes in alpha amplitude predict visual discrimination accuracy. While the alpha rhythm has 
long been known to play a significant role in voluntary attention, the present results demonstrate for the first time that the involuntary orienting of attention produced by an auditory cue also causes lateralized desynchronization of alpha activity over visual cortex that is directly related to visual discrimination performance. It appears that cue-induced alpha desynchronization over the occipital cortex is a manifestation of a general priming mechanism that improves visual processing, and this mechanism can be activated either by the voluntary or involuntary orienting of attention. The specific pattern of alpha modulations preceding correct and incorrect target discriminations suggests that involuntary orienting to the non-predictive sound has a rapid and purely facilitatory influence on processing targets on the cued side, with no inhibitory influence on targets on the opposite side. This lack of an early inhibitory influence may represent a fundamental difference between involuntary orienting and voluntarily directed attention, which reportedly involves a mixture of attended-side facilitation and unattended-side suppression that develop over longer cue-target intervals.

\section{ACKNOWLEDGMENTS}

This research was supported by Natural Science Foundation of China 31400868 (W.F.F), the U.S. National Science Foundation Grant BCS-1029084 (S.A.H.), the Natural Sciences and Engineering Research Council of Canada and the Canada Foundation for Innovation (J.J.M.), and Marie Curie fellowship EU Grant PIOF-GA-2012-329920 (V.S.S).

\section{REFERENCES}


Banerjee, S., Synder, A.C., Molholm, S., and Foxe, J.J. 2011. Oscillatory alpha-band mechanisms and the deployment of spatial attention to anticipated auditory and visual target locations: supramodal or sensory-specific control mechanisms. J. Neurosci. 31(27), 9923-9932.

Briand, K.A., 1998. Feature integration and spatial attention: more evidence of a dissociation between endogenous and exogenous orienting. J. Exp. Psychol.: Hum. Percept. \& Perform. 24(4), 1243-1256.

Calvert, G.A., Spence, C., Stein, B.E., 2004. The handbook of multisensory processes. MIT Press, Cambridge, MA.

Cheal, M., Lyon, D.R., 1991. Central and peripheral precuing of forced-choice discrimination. Q. J. Exp. Psychol. A. 43(4), 859-880.

Chen, X., Chen, Q., Gao, D., Yue, Z. 2012. Interaction between endogenous and exogenous orienting in crossmodal attention. Scand. J. Psychol. 53(4), 303-308.

Corbetta, M., Shulman, G. L., 2002. Control of goal-directed and stimulus-driven attention in the brain. Nat. Rev. Neurosci. 3(3), 201-215.

Cox, R.W., 1996. AFNI: software for analysis and visualization of functional magnetic resonance neuroimages. Comput. Biomed. Res. 29, 162-173.

Delorme, A., Makeig, S., 2004. EEGLAB: an open source toolbox for analysis of single-trial EEG dynamics including independent component analysis. J. Neurosci. Methods 134, 9-21.

Dufour, A., 1999. Importance of attentional mechanisms in audiovisual links. Exp. Brain Res. $126,215-222$. 
Ergenoglu, T., Demiralp, T., Bayraktaroglu, Z., Ergen, M., Beydagi, H., Uresin, Y., 2004. Alpha rhythm of the EEG modulates visual detection performance in humans. Cogn. Brain Res. 20, $376-383$.

Feng, W., Störmer, V.S., McDonald, J.J., Martinez, A., Hillyard, S.A., 2014. Sounds activate visual cortex and improve visual discrimination. J. Neurosci. 34, 9817-9824.

Foxe, J.J., Snyder, A.C., 2011. The role of alpha-band brain oscillations as a sensory suppression mechanism during selective attention. Front. Psychol. 2,154. doi:10.3389/fpsyg.2011.00154.

Gould, I.C., Rushworth, M.F., Nobre, A.C., 2011. Indexing the graded allocation of visuospatial attention using anticipatory alpha oscillations. J. Neurophysiol. 105, 1318-1326.

Grave de Peralta Menendez, R., Murray, M.M., Michel, C.M., Martuzzi, R., Gonzalez Andino, S.L., 2004. Electrical neuroimaging based on biophysical constraints. NeuroImage 21, 527-539.

Green, J.J., McDonald, J.J., 2010. Brief Reports: The role of temporal predictability in the anticipatory biasing of sensory cortex during visuospatial shifts of attention. Psychophysiol. 47, $1057-1065$.

Harter, M.R., Miller, S.L., Price, N.J., Lalonde, M.E., Keyes, A.L., 1989. Neural processes involved in directing attention. J. Cogn. Neurosci. 1, 223-237.

Hanslmayr, S., Aslan, A., Staudigl, T., Klimesch, W., Herrmann, C.S., Bäuml, K.H., 2007. Prestimulus oscillations predict visual perception performance between and within subjects. NeuroImage 37, 1465-1473.

Händel, B.F., Haarmeier, T., Jensen, O., 2011. Alpha oscillations correlate with the successful inhibition of unattended stimuli. J. Cogn. Neurosci. 23, 2494-2502. 
Hopf, J.M., Mangun, G.R., 2000. Shifting visual attention in space: An electrophysiological analysis using high spatial resolution mapping. Clin. Neurophysiol. 111, 1241-1257.

Hillyard, S.A., Störmer, V.S., Feng, W., Martinez, A., McDonald, J.J., 2016. Cross-modal orienting of visual attention. Neuropsychologia, 83, 170-178.

Hanslmayr, S., Gross, J., Klimesch, W., Shapiro, K.L., 2011. The role of alpha oscillations in temporal attention. Brain Res. Rev. 67, 331-343.

Ikkai, A., Dandekar, S., Curtis, C.E., 2016. Lateralization in alpha-band oscillations predicts the locus and spatial distribution of attention. Plos ONE. 11(5),e0154796, doi:10.1371/journal.

Jensen, O., Mazaheri, A., 2010. Shaping functional architecture by oscillatory alpha activity: gating by inhibition. Front. Hum. Neurosci. 4,186. doi:10.3389/fnhum.2010.00186.

Jonides, J., 1980. Voluntary versus automatic control over the mind's eye's movement. In: Long, J.B., Baddely, A. (Eds.), Attention and performance IX. Hillsdale, NJ: Erlbaum.

Kelly, S.P., Lalor, E.C., Reilly, R.B., Foxe, J.J., 2006. Increases in alpha oscillatory power reflect an active retinotopic mechanism for distracter suppression during sustained visuospatial attention. J. Neurophysiol. 95, 3844-3851.

Kelly, S.P., Gomez-Ramirez, M., Foxe, J.J., 2009. The strength of anticipatory spatial biasing predicts target discrimination at attended locations: A high-density EEG study. Eur. J. Neurosci. $30,2224-2234$.

Kincade, J.M., Abrams, R.A., Astafiev, S.V., Shulman, G.L., Corbetta. M., 2005. An eventrelated functional magnetic resonance imaging study of voluntary and stimulus-driven orienting of attention. J. Neurosci. 25(18), 4593-604 
Klimesch, W., 2012. Alpha-band oscillations, attention, and controlled access to stored information. Trends Cogn. Sci. 16, 606-617.

Koelewijn, T., Bronkhorst, A., Theeuwes, J. 2009. Auditory and visual capture during focused visual attention. J. Exp. Psychol. Hum. Percept. Perform. 35(5), 1303-1315.

Lopez-Calderon, J., Luck, S.J., 2014. ERPLAB: an open-source toolbox for the analysis of event-related potentials. Front. Hum. Neurosci. 8,213. doi:10.3389/fnhum.2014.00213.

Mayers, A.R., Dorflinger, J.M., Rao, S.M., Seidenberg, M., 2004. Neural networks underlying endogenous and exogenous visual spatial orienting. NeuroImage 23, 534-541.

Mazaheri. A., Jensen, O., 2008. Asymmetric amplitude modulations of brain oscillations generate slow evoked responses. J. Neurosci. 28, 7781-7787.

Mazaheri, A., van Schouwenburg, M.R., Dimitrijevic, A., Denys, D., Cools, R., Jensen, O., 2014. Region-specific modulations in oscillatory alpha activity serve to facilitate processing in the visual and auditory modalities. NeuroImage $87,356-362$.

Mercier, M.R., Foxe, J.J., Fiebelkorn, I.C., Butler, J.S., Schwartz, T.H., Molholm, S., 2013. Auditory-driven phase reset in visual cortex: human electrocorticography reveals mechanisms of early multisensory integration. NeuroImage 79, 19-29.

Mlynarski, W., Freigang, C., Bennemann, J., Stohr, M., Rubsamen, R., 2014. Position of acoustic stimulus modulates visual alpha activity. NeuroReport 25,833-837.

Murray, M.M., Wallace, M., 2012. The neural bases of multisensory processes. Boca Raton (FL): CRC Press. 
Müller, H.J., Rabbitt, P.M.A., 1989. Spatial cueing and the relation between the accuracy of "where" and "what" decisions in visual search. Q. J. Exp. Psychol. A 41, 747-773.

McDonald, J.J., Whitman, J.C., Störmer, V.S., Hillyard, S.A., 2013a. Involuntary cross-modal spatial attention influences visual perception. In: Mangun, G.R. Cognitive Electrophysiology of Attention. (pp:82-94). Amsterdam, Elsevier.

McDonald, J.J., Ward, L.M., 2000. Involuntary listening aids seeing: Evidence from human electrophysiology. Psychol. Sci. 11,167-171.

McDonald, J.J., Teder-Sälejärvi, W.A., Hillyard, S.A., 2000. Involuntary orienting to sound improves visual perception. Nature 407, $906-908$.

McDonald, J.J., Teder-Sälejärvi, W.A., Di Russo, F., Hillyard, S.A., 2005. Neural basis of auditory-induced shifts in visual time order perception. Nat. Neurosci. 8, 1197-1202.

McDonald, J.J., Störmer, V.S., Martinez, A., Feng, W., Hillyard, S.A., 2013b. Salient sounds activate visual cortex automatically. J. Neurosci. 33, 9194-9201.

McDonald, J.J., Green, J.J., Störmer, V.S., Hillyard, S.A., 2012. Cross-modal spatial cueing of attention influences visual perception. In: Murray, M.M., Wallace, M.T. (Eds.), Frontiers in the neural bases of multisensory processes, Boca Raton, FL: CRC, pp. 509-527,

McDonald, J.J., Teder-Sälejärvi, W.A., Di Russo, F., Hillyard, S.A., 2003. Neural substrates of perceptual enhancement by cross-modal spatial attention. J. Cogn. Neurosci. 15,10-19.

McCarthy, G., Wood, C.C., 1985. Scalp distributions of event-related potentials: An ambiguity associated with analysis of variance models. Electroencephalogr. Clin. Neurophysiol. 62, 203208. 
Nakayama, K., Mackeben, M., 1989. Sustained and transient components of focal visual attention. Vision Res. 29, 1631-1647.

Nobre, A.C., Sebestyen, G.N., and Miniussi, C., 2000. The dynamics of shifting visuospatial attention revealed by event-related brain potentials. Neuropsychologia 38, 964-974.

Okazaki, Y.O., De Weerd, P., Haegens, S., Jensen, O., 2014. Hemispheric lateralization of posterior alpha reduces distracter interference during face matching. Brain Res. 1590, 56-64.

Payne, L., Guillory, S., Sekuler, R., 2013. Attention-modulated alpha-band oscillations protect against intrusion of irrelevant information. J. Cogn. Neurosci. 25, 1463-1476.

Payne, L., Sekuler, R., 2014. The importance of ignoring Alpha Oscillations protect selectivity. Curr. Dir. Psychol. Sci. 23, 171-177.

Posner, M.I., Nissen, M.J., Klein, R.M., 1976. Visual dominance: an information-processing account of its origins and significance. Psychol. Rev. 83(2), 157-171.

Posner, M.I., Cohen, Y., 1984. Components of visual orienting. In: Bouma, H., Bouwhuis, D. (Eds.), Attention and Performance, vol. X. Lawrence Erlbaum, London, pp. 531-554.

Rihs, T.A., Michel, C.M., Thut, G., 2007. Mechanisms of selective inhibition in visual spatial attention are indexed by a-band EEG synchronization. Eur. J. Neurosci. 25, 603-610.

Rihs, T.A., Michel, C.M., Thut, G., 2009. A bias for posterior $\alpha$-band power suppression versus enhancement during shifting versus maintenance of spatial attention. NeuroImage 44, 190-199

Rorden, C., Brett, M., 2000. Stereotaxic display of brain lesions. Behav. Neurol. 12, 191-200. 
Romei, V., Brodbeck, V., Michel, C., Amedi, A., Pascual-Leone, A., Thut, G., 2008.

Spontaneous fluctuations in posterior alpha-band EEG activity reflect variability in excitability of human visual areas. Cereb. Cortex 18, 2010-2018.

Romei, V., Gross, J., Thut, G., 2012. Sounds reset rhythms of visual cortex and corresponding human visual perception. Curr. Biol. 22, 807-813.

Santangelo, V., Olivetti Belardinelli, M., Spence, C., Macaluso, E. 2009. Interactions between voluntary and stimulus-driven spatial attention mechanisms across sensory modalities. J. Cogn. Neurosci. 21(12), 2384-2397.

Snyder, A.C., Foxe, J.J., 2010. Anticipatory attentional suppression of visual features indexed by oscillatory alpha-band power increases: a high-density electrical mapping study. J. Neurosci. 30, 4024-4032.

Sauseng, P., Klimesch, W., Stadler, W., Schabus, M., Doppelmayr, M., Hanslmayr, S., Gruber, W.R., Birbaumer, N., 2005. A shift of visual spatial attention is selectively associated with human EEG alpha activity. Eur. J. Neurosci. 22, 2917-2926.

Spence, C., Driver, J., 1997. Audiovisual links in exogenous covert spatial orienting. Percept. Psychophys. 59, 1-22.

Spence, C., Driver, J., 2004. Crossmodal space and crossmodal attention. Oxford: Oxford UP. Störmer, V.S., McDonald, J.J., Hillyard, S.A., 2009. Cross-modal cueing of attention alters appearance and early cortical processing of visual stimuli. Proc. Natl. Acad. Sci. USA 106, 22456-22461. 
Störmer, V.S., Feng, W., McDonald, J.J., Martinez, A., Hillyard, S.A., 2016. Salient sounds elicit slow potential shifts paralleled by alpha rhythm desynchronization in contralateral visual cortex. J. Cogn. Neurosci. 28(3), 433-445.

Talairach, J., Tournoux, P., 1988. Co-planar stereotaxic atlas of the human brain: 3-dimensional proportional system: an approach to cerebral imaging. New York: Thieme.

Thut, G., Miniussi, C., and Gross, J., 2012. The functional importance of rhythmic activity in the brain. Curr. Biol. 22,R658-R663.

Thut, G., Nietzel, A., Brandt, S.A., Pascual-Leone, A., 2006. Alpha-band electroencephalographic activity over occipital cortex indexes visuospatial attention bias and predicts visual target detection. J. Neurosci. 26, 9494-9502.

Trenner, M.U., Heekeren, H.R., Bauer, M., Rössner, K., Wenzel, R., Villringer, A., Fahle, M., 2008. What happens in between? Human oscillatory brain activity related to crossmodal spatial cueing. PLoS ONE 3,e1467. doi:10.1371/journal.pone.0001467

van Dijk, H., van der Werf, J., Mazaheri, A., Medendorp, W.P., Jensen, O., 2010. Modulations in oscillatory activity with amplitude asymmetry can produce cognitively relevant event-related responses. Proc. Natl. Acad. Sci. USA 107, 900-905.

van Dijk, H., Schoffelen, J.M., Oostenveld, R., Jensen, O., 2008. Prestimulus oscillatory activity in the alpha band predicts visual discrimination ability. J. Neurosci. 28,1816-1823.

Worden, M.S., Foxe, J.J., Wang, N., Simpson, G.V., 2000. Anticipatory biasing of visuospatial attention indexed by retinotopically specific alpha-band electroencephalography increases over occipital cortex. J. Neurosci. 20,RC63. 
Wright, R.D., Ward, L.M., 2008. Orienting of attention. New York: Oxford University Press.

Yamagishi, N., Goda, N., Callan, D.E., Anderson, S.J., Kawato, M., 2005. Attentional shifts

towards an expected visual target alter the level of alpha-band oscillatory activity in the human calcarine cortex. Cogn. Brain Res. 25,799-809. 


\section{FIGURE LEGENDS}

Figure 1. Experimental design: Illustration of the stimulus sequence. An example of a validly cued trial is shown.

Figure 2. ACOP waveforms and time frequency plots of alpha desynchronization on cue-only trials.

(A) ACOP waveforms elicited on sound-only (cue-only) trials. The waveforms were averaged over 6 homologous pairs of posterior electrodes, separately for sites ipsilateral and contralateral to the sound cue. Shaded area (200-400 ms after cue onset) shows the interval where ACOP was measured. Head maps show the topographical distribution of the ACOP, defined as the contralateral minus ipsilateral difference amplitude over two successive time intervals, projected onto the right hemisphere. The six electrode sites indicated with bolder dots are those used in the quantitative analyses of ACOP.

(B) Time-frequency plot of lateralized alpha desynchronization (contralateral minus ipsilateral amplitudes) following the auditory cue was obtained from the same electrode sites as the ACOP. Head maps show the scalp topography of the contralateral minus ipsilateral alpha-band (8-14 Hz) desynchronization to be similar to that of the ACOP. The six electrode sites indicated with bolder dots are those used in the quantitative analyses of alpha desynchronization.

Figure 3. ACOP waveforms and time frequency plots of alpha desynchronization on different types of trials.

(A) ACOP waveforms and time frequency plot of concurrent lateralized alpha desynchronization (averaged over 6 homologous pairs of posterior electrodes) on trials where validly cued targets were correctly discriminated. The time-frequency plot shows contralateral minus ipsilateral difference amplitudes for different EEG frequencies. 
(B) No significant ACOP or lateralized alpha desynchronization was evident on validly cued trials when the target was not correctly discriminated.

(C\&D) The ACOP and lateralized occipital alpha desynchronization were both evident on invalidly cued trials, both for trials having correct (C) and incorrect (D) target discriminations, but with lesser amplitudes than in the case of the validly cued-correct trials shown in (A).

Figure 4. The topographical distribution maps of ACOP and lateralized alpha $(8-14 \mathrm{~Hz})$ desynchronization (contralateral minus ipsilateral difference amplitudes), projected onto the right hemisphere. Maps are shown separately for four different types of trials (A-D), and both measures were plotted separately over 200-300 ms and 300-400 ms intervals.

Figure 5. Mean amplitudes of ACOP and lateralized alpha desynchronization (8-14 Hz) elicited by the sound cue for different types of trials.

Both the ACOP (left bars) and the lateralized occipital alpha desynchronization (right bars) were measured within the time windows of (A) 200-300 ms and (B) 300-400 ms after cue onset, averaged over 6 occipital electrodes for each type of trial. Note that both the ACOP and the lateralized occipital alpha desynchronization were much greater on correct trials than incorrect trials for validly cued targets within both time intervals, but not for invalidly cued targets. Error bars represent standard errors of the means. Significance levels for the correct vs. incorrect comparisons: $* \mathrm{p}<.05, * * \mathrm{p}<.01, * * * \mathrm{p}<.001$

Figure 6. Correlations between the amplitudes of alpha desynchronization and the ACOP on different types of trials.

Scatter plots showing between-subject correlations between the amplitudes of lateralized (contralateral minus ipsilateral) occipital alpha desynchronization and the ACOP on valid correct, valid incorrect, invalid correct, and invalid incorrect trials. Neither alpha desynchronization nor 
ACOP was evident on the valid incorrect trials. Amplitude measures were made over the interval of 200-400 ms after cue onset and averaged over 6 occipital electrodes.

Figure 7. Amplitudes of occipital alpha desynchronization elicited by sounds (averaged over the 200-400ms post-cue interval) plotted separately for hemispheres contralateral and ipsilateral with respect to the sound's location. Significant alpha desynchronization was observed over both contralateral and ipsilateral hemispheres for all eight validity $\mathrm{x}$ accuracy conditions. Note: $* *=p$ $<.001$.

Figure 8. Source localizations of the ACOP and lateralized (contralateral minus ipsilateral) alpha desynchronization. The estimated sources for ACOP and lateralized alpha desynchronization were obtained by LAURA for the three different types of trials derived from the contralateral minus ipsilateral difference maps (left column, ACOP; right column, alpha desynchronization). Sources are projected on a standard structural brain image. Neither alpha desynchronization nor ACOP was evident on the valid incorrect trials. 


\section{Experimental design}

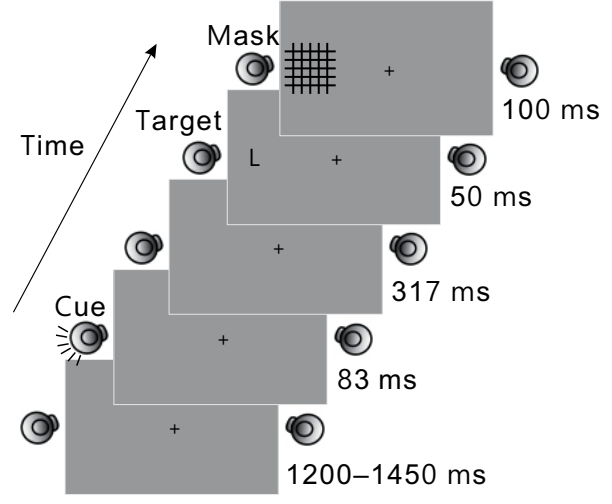

Figure1_R1 
A ACOP for cue-only trials
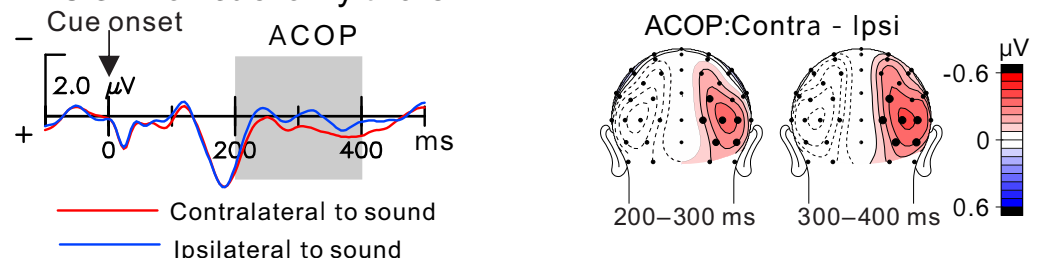

B Time-frequency for cue-only trials
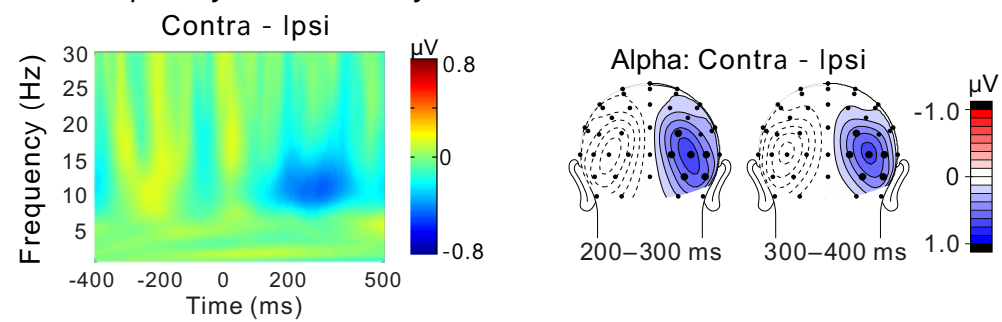

Figure2_R1 


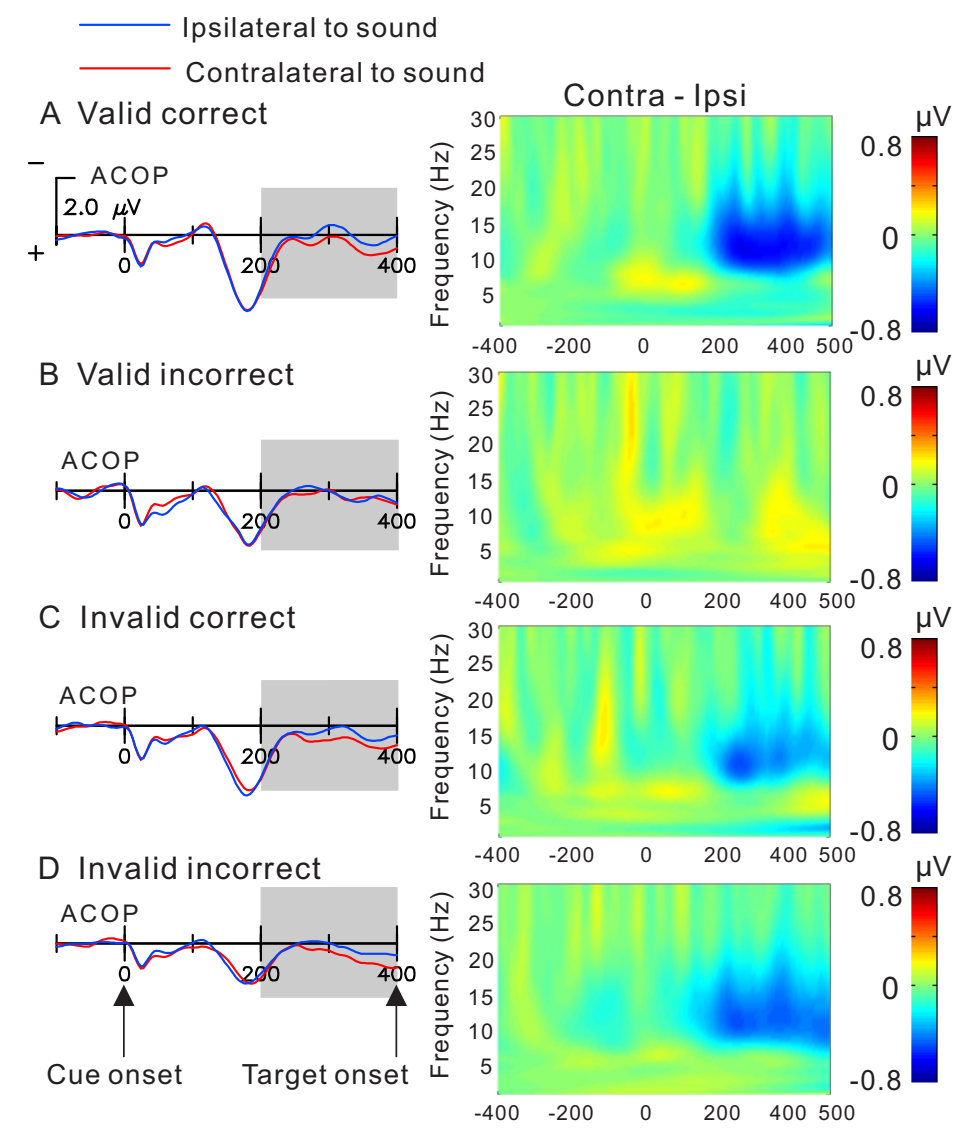

Figure3_R1 


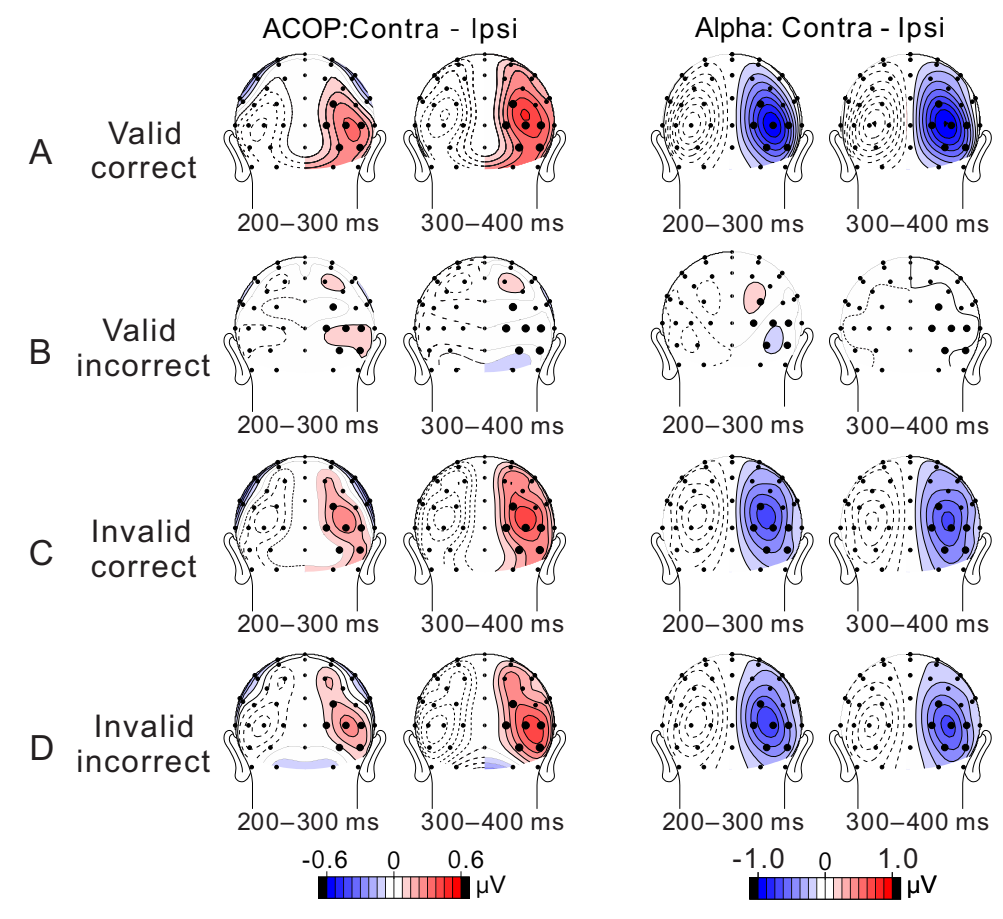

Figure4_R1 
A ACOP and alpha desynchronization in $200-300 \mathrm{~ms}$ ACOP: $200-300 \mathrm{~ms}$

Alpha: $200-300 \mathrm{~ms}$
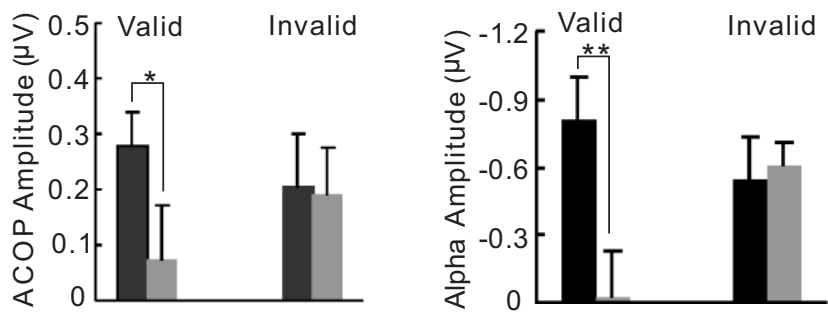

Correct Incorrect

B ACOP and alpha desynchronization in $300-400 \mathrm{~ms}$

ACOP: $300-400 \mathrm{~ms}$

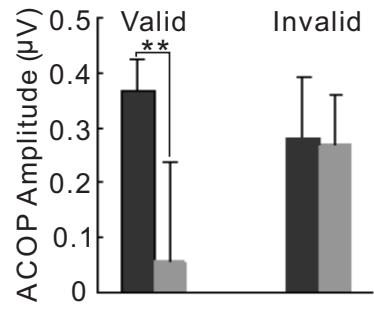

Alpha: $300-400 \mathrm{~ms}$

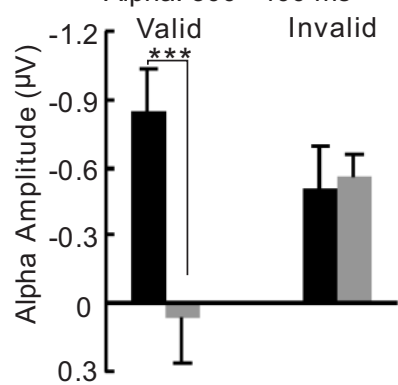

Figure5_R2 

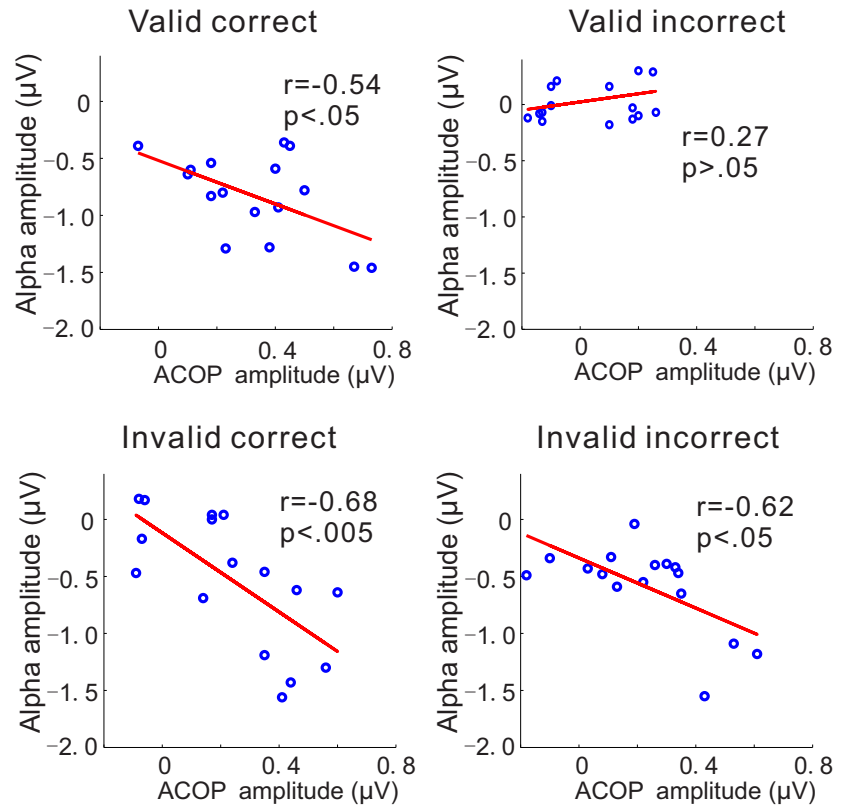

Figure6_R1 
Alpha amplitudes over $200-400 \mathrm{~ms}$

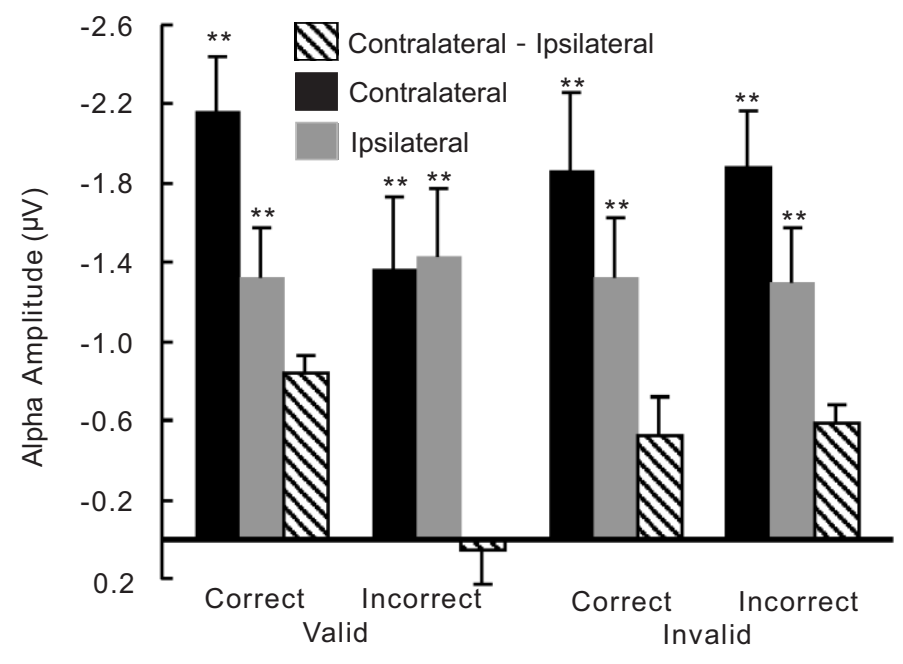

Figure7_R1 
A Valid correct
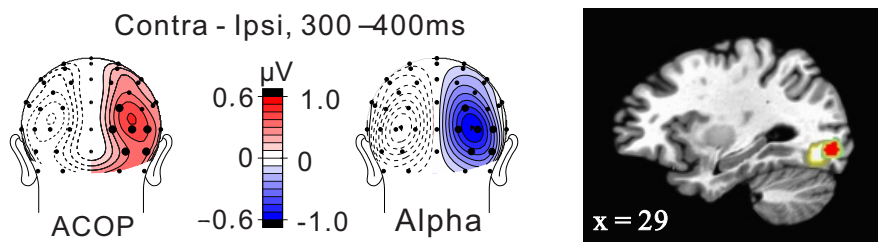

B Invalid correct
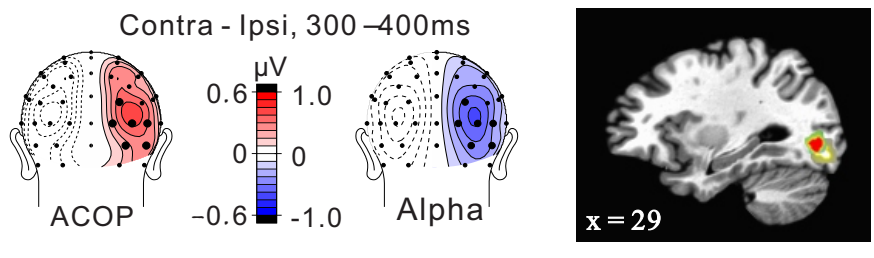

C Invalid incorrect
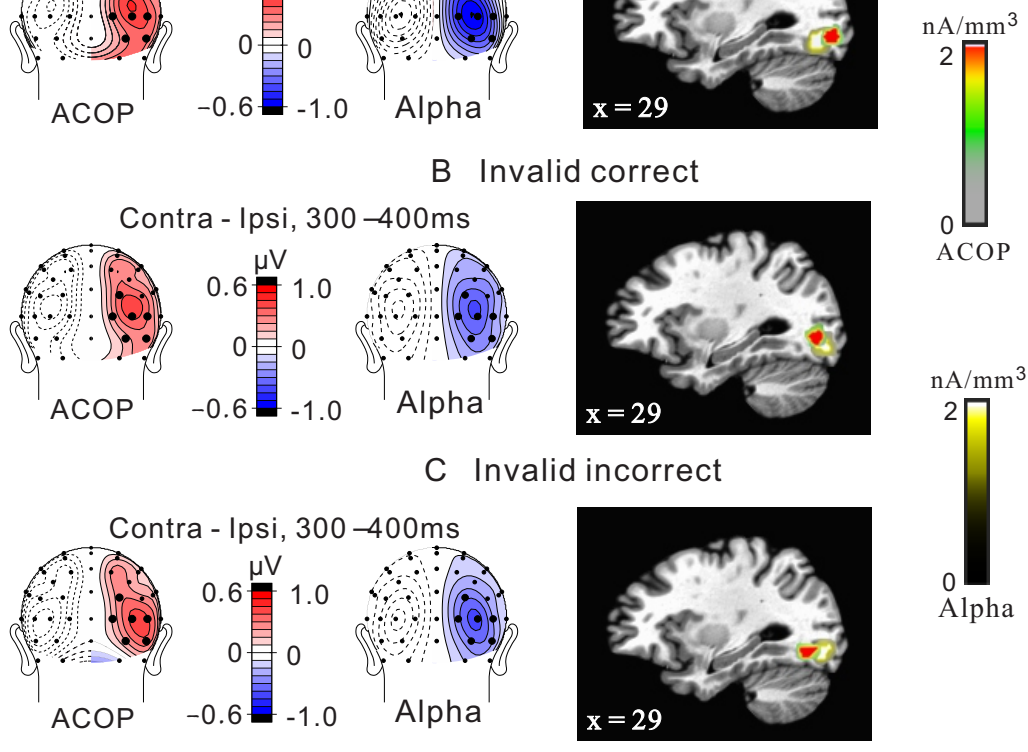

Figure8_R1 